E-mail: jzus@zju.edu.cn

\title{
Editorial:
}

\section{Data-driven digital entertainment: a computational perspective}

Yue-ting ZHUANG

\author{
Guest Editor-in-Chief for this special issue \\ Zhejiang University, Hangzhou 310027, China \\ E-mail: yzhuang@cs.zju.edu.cn
}

doi: 10.1631 jzus.CIDE1300

Today massive collections of data can be obtained across different sources (or domains), e.g., the depth data from Kinect, the geometrical data from scanning devices, the imagery/video data from cameras, and the motion data from mocap devices. Since heterogeneous data may have different discriminative powers and are intrinsically complementary for certain tasks, it is desirable to leverage all the information available in digital entertainment. For example, the acquired 3D geometry and texture are jointly exploited to construct the colored 3D environment models; high-resolution geometry and motioncaptured data are obtained to synthesize and re-target facial animations; both visual and acoustical features are contextually applied to classification. Therefore, it poses a significant challenge for the appropriate utilization of the different varieties of heterogeneous data in digital entertainment.

The intuition behind this challenge can be considered from a data-driven computational perspective. The underlying formalism of the data-driven computation is to derive mathematical expressions directly from the database with a minimum of a priori hypotheses. The typical approaches of data-driven computation are generative models (e.g., Gaussian mixture model, hidden Markov model, Naive Bayes, and latent Dirichlet allocation) and discriminative models (e.g., support vector machines, boosting, conditional random fields, and neural networks). Generative models are desirable when they capture

C Zhejiang University and Springer-Verlag Berlin Heidelberg 2013 the generation process of a data population of interest. However, it is usually difficult to learn a generative model for the data in a high-dimensional setting since the structure of the data space is hard to be introduced into the generative model due to the unsupervised manner. Discriminative models tend to discover a distinctive representation of the data and typically have a strong discriminative power. However, their modeling capabilities may be limited when the focus is on obtaining a clearly separable boundary between classes of the data, resulting in a less powerful capability to create (synthesize) new samples of interest.

Driven by the emerging large scale heterogeneous data and their embedding priors (such as the intrinsic structures and the contextual correlations in the data), we have witnessed a rapid development of the data-driven computation in digital entertainment, intelligent $\mathrm{CAD}$, and digital art. Undoubtedly, the convergence of different research fields (e.g., computer vision, machine learning, and computer graphics) and the resulting technologies from the research in these fields will lead to a better modeling of 3D environments, a more realistic synthesis of facial animations, and a better recognition performance. In fact, how to develop a more interpretable data-driven computational model still remains a challenge. We are confident that an interpretable computational model certainly benefits from theoretical research and real-world applications in digital entertainment.

Motivated by the above considerations, we will hold the 10th National Conference on Intelligent CAD and Digital Entertainment (CIDE 2013) and the 8th National Conference on Digital Entertainment and Art (DEA 2013) in Zhenjiang, Jiangshu, China, from August 15 to August 16, 2013. CIDE 2013 and DEA 2013 will again bring together the researchers, developers, practitioners, and educators in the field of digital entertainment, intelligent $\mathrm{CAD}$, art, as well as other related areas. Both theoretical breakthroughs and practical systems will be reported at the 
conferences. This special issue selects 10 best papers from the submissions to CIDE 2013 and DEA 2013. The topics in this special issue cover both algorithmic development and technical surveys.

Algorithmic papers consist of the synthesis of 3D models (Song et al., 2013), portrait drawing (Wang et al., 2013), facial animation (Xu et al., 2013), extraction of the feature lines (Zhang et al., 2013), face recognition (Ma et al., 2013), and speech emotion recognition (Mao et al., 2013). Four survey papers focus on the recent advance and future challenge of the related research, including the surveys of crowd animation (He et al., 2013), structure from motion (Wei et al., 2013), high-dimensional indexing technologies (Ai et al., 2013), and object representation with local features (Cao et al., 2013).

We graciously acknowledge the reviewing effort of the program committee members. The many useful comments provided in the reviews are useful to improve the quality of the papers.

We also greatly appreciate the support from Helen (Y.H.) Zhang, managing editor of Journals of Zhejiang University-SCIENCE $(A / B / C)$. I also would like to thank Zi-yang Zhai, editor of this special issue, for the helpful guidance and constructive feedback in the entire process of helping organize and publish this special issue.

\section{References}

Ai, L.F., Yu, J.Q., He, Y.F., Guan, T., 2013. High-dimensional indexing technologies for large scale content-based image retrieval: a review. J. Zhejiang Univ.-Sci. C (Comput. \& Electron.), 14(7):505-520. [doi:10.1631/jzus.CIDE1304]

Cao, J., Mao, D.H., Cai, Q., Li, H.S., Du, J.P., 2013. A review of object representation based on local features. $J$. Zhejiang Univ.-Sci. C (Comput. \& Electron.), 14(7):495504. [doi:10.1631/jzus.CIDE1303]

He, G.Q., Yang, Y., Chen, Z.H., Gu, C.H., Pan, Z.G., 2013. A review of behavior mechanisms and crowd evacuation animation in emergency exercises. J. Zhejiang Univ.-Sci. C (Comput. \& Electron.), 14(7):477-485. [doi:10.1631/ jzus.CIDE1301]

Ma, X.H., Tan, Y.Q., Zheng, G.M., 2013. A fast classification scheme and its application to face recognition. J. Zhejiang Univ.-Sci. C (Comput. \& Electron.), 14(7):561-572. [doi:10.1631/jzus.CIDE1309]

Mao, Q.R., Zhao, X.L., Huang, Z.W., Zhan, Y.Z., 2013. Speaker-independent speech emotion recognition by fusion of functional and accompanying paralanguage features. J. Zhejiang Univ.-Sci. C (Comput. \& Electron.), 14(7):573-582. [doi:10.1631/jzus.CIDE1310]

Song, M.F., Sun, Z.X., Zhang, Y., Zhang, F.Q., 2013. Synthesis of 3D models by Petri net. J. Zhejiang Univ.-Sci. C (Comput. \& Electron.), 14(7):521-529. [doi:10.1631/jzus. CIDE1305]

Wang, L., Lou, L.M., Yang, C.L., Huang, Y.Z., Meng, X.X., 2013. Portrait drawing from corresponding range and intensity images. J. Zhejiang Univ.-Sci. C (Comput. \& Electron.), 14(7):530-541. [doi:10.1631/jzus.CIDE1306]

Wei, Y.M., Kang, L., Yang, B., Wu, L.D., 2013. Applications of structure from motion: a survey. J. Zhejiang Univ.-Sci. C (Comput. \& Electron.), 14(7):486-494. [doi:10.1631/ jzus.CIDE1302]

Xu, S., Ma, G., Meng, W., Zhang, X., 2013. Statistical learning based facial animation. J. Zhejiang Univ.-Sci. C (Comput. \& Electron.), 14(7):542-550. [doi:10.1631/jzus.CIDE 1307]

Zhang, Y.Y., Sun, Z.X., Liu, K., Song, M.F., Zhang, F.Q., 2013. Extracting 3D model feature lines based on conditional random fields. J. Zhejiang Univ.-Sci. C (Comput. \& Electron.), 14(7):551-560. [doi:10.1631/jzus.CIDE1308]

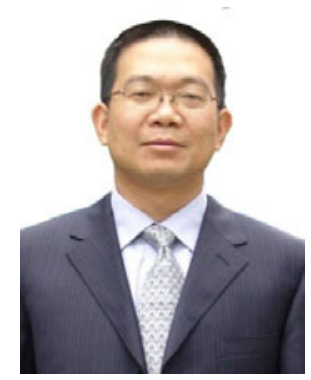

Yue-ting Zhuang, Associate Editor-in-Chief of $J Z U S-C$, is a professor and Dean of the College of Computer Science, Zhejiang University. His research interests include artificial intelligence, multimedia retrieval, computer animation, and digital library. He has won various honors such as the Award of National Science Fund for Distinguished Young Scholars of China (2005), the Cheung Kong professor (appointed by the Ministry of Education of China in 2008), the Chief Scientist of the National Basic Research Program of China (973) in 2012. $\mathrm{He}$ is on the editorial board of Science in China Series F: Information Science. He is director of MOE-Microsoft Key Laboratory of Visual Perception, Zhejiang University. He currently serves as the Deputy President of the Chinese Society of Image and Graphics. From February, 1997 to August, 1998, Yue-ting Zhuang was a visiting scholar at University of Illinois at Urbana-Champaign. 